УДК 004.891.3; 621.327;519.2; 534.8

V.N. Zvaritch, Dr Sc. (Eng.), M.V. Myslovych, Dr Sc. (Eng.)

Institute of Electrodynamics of the National

Academy of Sciences of Ukraine

(56, Pobeda Av. Kyiv, 03680, Ukraine,

e-mail: zvaritch@nas.gov.ua; mysl@ied.org.ua)

\title{
White Noise in Some Simulation Problems of Information Signals*
}

A constructive method of information signal mathematical models characterization on the white noise basis is developed. Linear random processes, linear random processes with periodic structures, linear autoregressive processes, linear autoregressive processes with periodic structures are represented as examples of the method application.

Ke y w ords: white noise, linear random process, linear random process with periodic structures, linear autoregressive processes.

Introduction. Many information signals like vibrations, acoustic emission signals, control signals, etc., can be represented as a response of some linear system to the white noise action [1-6]. Now we try to make the white noise models more exact and make more detailed mathematical descriptions, definitions, and practical applications of the white noise processes. The first detailed investigation of white noise processes was made by K. Ito in 1954 [1]. However, the beginning of the white noise theory can be traced back to the 1930's to the works of A. Kolmogorov [2] and A. Khinchin [3], where the processes with independent increments closely related to white noise were considered.

White noise definition. In the most simple case, in technical applications, white noise can be defined as a generalized process with noncorrelated values $\{\varsigma(t), t \in T\}$ such that [7]

$$
M[\varsigma(t)]=0, M[\varsigma(t) \varsigma(t+\tau)]=\kappa_{2} \delta(\tau),
$$

where $\delta(\tau)$ is a Dirac delta function. The stochastic process is generalized and

\footnotetext{
* This work was supported by the National Academy of Sciences of Ukraine under grants in the framework of OBJEDNANNYA-3 and RESURS-2 programs.
}

(c) V.N. Zvaritch, M.V. Myslovych, 2018 
stationary. The correlation function of the process is also a generalized one and is determined as

$$
R(\tau)=\kappa_{2} \delta(\tau), \tau \in T,
$$

where $\kappa_{2}>0$ is an intensity of the white noise. In the case of the nonstationary white noise, the parameter $\kappa_{2}$ will depend on time $t$. White noise allows a spectral decomposition

$$
\zeta(t)=\frac{1}{2 \pi} \int_{-\infty}^{\infty} e^{i \omega t} d z(\omega),
$$

where $z(\omega)$ is a process with noncorrelated increments.

The infinitesimal "elementary oscillations" $e^{i \omega t} d z(\omega)$ have equal infinitesimal mean power and are mutually noncorrelated at any frequencies $(\omega)$ because of noncorrelated increments of the process $z(\omega)$. The mean square of their infinitesimal amplitude has a mathematical expectation $M|d z(\omega)|^{2}=$ $=\kappa^{2} d \omega, \omega \in(-\infty, \infty)$.

The Gilbert stochastic process with representation (2) is called Loeve harmonizable process. However, the white noise cannot be considered as the Loeve harmonizable stochastic process, because it is not a Gilbert process: it does not have finite variance or finite mean power. Nevertheless, in a general sense, the white noise can be considered as a harmonizable one. Then, its generalized harmonizable correlation function can be derived from (2) and is determined as

$$
R(\tau)=\frac{1}{2 \pi} \int_{-\infty}^{\infty} \kappa_{2} e^{-i \omega t} d(\omega) .
$$

Thus, the white noise with continuous time has constant spectral power density at infinite frequency bound and equals $S(\omega)=\kappa_{2}, \omega \in(-\infty, \infty)$, i.e., the power of every infinitesimal harmonic component equals $\kappa_{2} d \omega$.

The general power of all these harmonic components at any finite frequency bound is proportional to the value of the latter and equals infinity at the infinite frequency bound. Therefore the white noise with continuous time is not physically possible. White noise processes with continuous time are closely related to those with independent increments, which even in the case of continuous time can be considered as non-generalized processes.

A process with independent increments is one whose random variables $\left\{\eta\left(t_{n}\right)\right\}$ have the property that, if $t_{1}<\ldots<t_{n}(n \geq 3)$ the differences $\eta\left(t_{-m+1}\right)-\eta\left(t_{-m}\right), \ldots$ $\ldots, \eta\left(t_{0}\right)-\eta\left(t_{-1}\right), \ldots, \eta\left(t_{n}\right)-\eta\left(t_{n-1}\right), T \in(-\infty, \infty)$ are mutually independent.

The value of $\eta\left(t_{0}\right)$ is a limit value of the process $\eta(t)$, if $t_{0}=\min T>-\infty$ or $t_{0}=\max T<\infty$. It is this kind of processes with independent increments, which is 
generally considered in the theory of random processes [8]. For these processes $t_{0}$ is usually taken at a zero point, and $\eta(0)$ is considered to be of nonrandom value.

For the processes with continuous time the values of the white noise process in the strong sense and of the process with independent increments are connected by an equation

$$
\eta(t)=\int_{0}^{t} \varsigma(\tau) d \tau, t \in[0, \infty]
$$

where $\{\varsigma(t), t \in[0, \infty)\}$ is a white noise in the strong sense with continuous time. The increments $\eta(\tau)$ at the non-crossing intervals are independent. If there exists a difference limit for $\Delta^{s} \eta(\tau)=\eta(\tau)-\eta(s)$, i.e., $\varsigma_{\tau}=\lim _{|\tau-s| \rightarrow 0} \frac{\Delta^{s} \eta(\tau)}{\tau-s}$, then the limit process $\varsigma_{\tau}$ is the white noise process in the strong sense with continuous time. However, in a general sense, such a derivative does not exist and the white noise with continuous time can be considered as a generalized process.

In contrast to the white noise with continuous time, the processes with independent increments are physically possible. Homogeneous characteristic function of the process with independent increments $\{\eta(t), t \in T\}$ is determined as

$$
f_{\eta}(u, t)=M e^{i u \eta(t)} .
$$

The one-dimensional characteristic function $f(u ; s, \tau)$ of the increments $\Delta^{s} \eta(\tau)$ is determined as $f(u ; s, \tau)=M e^{i u \Delta^{s} \eta(t)}$. A remarkable feature of the characteristic function (3) of the homogeneous processes with independent increments is that the corresponding distribution function belongs to the class of infinitely divisible ones, as described by Levy. A canonical representation of the characteristic function in the Levy form is

$$
\ln f_{\eta}(u, t)=|t|\left\{i \mu \varepsilon u-\frac{\delta^{2}}{2} u^{2}+\int_{-\infty}^{\infty}\left[e^{i u \varepsilon x}-1-\frac{i u \varepsilon x}{1+x^{2}}\right] d L(x)\right\}, \quad t \in(-\infty, \infty),
$$

where $\mu$ and $\delta>0$ are some constants, $\varepsilon=\operatorname{sign} t L(x)$ is a Poisson jump spectrum in the Levy form. It is noteworthy that $f_{\eta}(u, t) \equiv f(u ; 0, t)$. The above representation of the characteristic function can be regarded as the main result of the theory of the white noise with continuous time. Unfortunately, it has not yet found a wide application.

As was mentioned, the white noise with continuous time is a generalized stochastic process and it is not described by a distribution function, since such a function does not exist. The notions of the Gaussian or Poisson white noise indi- 
cate that the integral between boundary time intervals has the corresponding Normal or Poisson distribution, respectively.

White noise forms the basis of linear stochastic processes. The latter can be considered as a result of a linear filtration of white noise processes of different distributions. An account of the theory of linear stochastic processes was given in $[7,9]$.

Linear random process. In our paper we discuss a problem of statistical simulation of such signals. The problem, in general wording, consists in obtaining a sequence of pseudo random values with the given probability characteristics. The proposed problem solving approach is based on the Linear Random Process Theory [4] and it can be considered to be a development of genera- ting process method [7]. At first we state some knowledge of the Theory, and classify the simulating processes. Then we define concretely the simulation problem statement and discuss a method of solving.

A linear random process (LRP) is a functional of the following form

$$
\xi(t)=\int_{-\infty}^{\infty} \varphi(\tau, t) d \eta(\tau), \quad t \in(-\infty, \infty),
$$

where $\varphi(\tau, t) \in L_{2}(-\infty, \infty)$ with respect to $\tau$ for all $t$ is a non-stochastic real Hilbert function; $\eta(\tau), \eta(0)=0, \tau \in(-\infty, \infty)$ is a Hilbert stochastically continuous random process with independent increments that is often called a generating process. While solving many problems including statistical simulation it is convenient to consider the LRP as a response of a linear filter with the impulse transient function $\varphi(\tau, t)$ to the action of the white noise $\eta^{\prime}(\tau)$. It is understood that $\eta^{\prime}(\tau)$ is a generalized derivative of the corresponding process with independent increments.

It is known [4] that any stochastically continuous process with independent increments can be represented as a sum of two stochastically independent components which may be not present simultaneously: Gaussian and Poisson. We call the components as processes of the Gaussian and Poison types. The first type contains the homogeneous (Wiener) and non-homogeneous Gaussian processes with independent increments. Simple Poisson processes, renewal processes and their linear combinations, generalized Poisson processes with independent increments belong to the second type. Being generated by each of the mentioned processes LRP possess some typical properties. This fact is taken as a principle of the classification. For example, it can be shown that, if the generating process is of the Gaussian type, the corresponding LRP is a Gaussian stochastic process. The Poisson type of generating process leads to LRP describing impulse currents. We call such processes the impulse LRP. 
The type of integral representation kernel $\varphi(\tau, t)$ also influences typically the probability properties of the resulting process. As has been mentioned above, the physical meaning of some forming filter's impulse transient function can be attached to the $\varphi(\tau, t)$. For example, the exponential kernel corresponds to the case of a low pass filtration, and the exponential cosine kernel corresponds to the band pass filtration case. By analogy with the forming filter's type we often call the corresponding LRP the $R C$ - and $R L C$-noises.

Using a characteristic function of a linear stochastic process, one can perform a full analysis of the output signals of linear systems: calculate moments and distribution functions, analyze connections between input and output characteristics of the linear circuit [7,9]. White noise and corresponding linear stochastic processes can be generalized for the multivariate cases, i.e., the cases of stochastic white fields and linear stochastic fields.

Linear random process with periodic structures. We have to deal with non-stationary random processes in applications to many cases. Investigations of the periodic correlated random processes structure and statistical parameter estimations, at the correlation theory level have been developed by Ogura [8]. Random periodic processes were discussed for the first time by Slutskiy [10]. These processes can be defined in the following way. A real random process $\xi(t)$, $t \in(\infty, \infty)$ is called the real periodic random process, according to Slutskiy, if the fixed number $T>0$ exists for the process $\xi(t)$, such that the finite dimension vectors $\left(\xi\left(t_{1}\right), \xi\left(t_{2}\right), \ldots, \xi\left(t_{n}\right)\right)$ and $\left(\xi\left(t_{1}+T\right), \xi\left(t_{2}+T\right), \ldots, \xi\left(t_{n}+T\right)\right)$ are stochastically equivalent, in a wide sense, for all whole numbers $n>0$, where $t_{1}, t_{2}, \ldots$ is a set of separability of the process $\xi(t)$.

The main purpose of this paper is to make the periodic non-stationary random process more exact in an infinitely divisible class by detailed mathematical investigation of the problems connected with the description, definition, and practical application of the process.

According to [11, 12], for the processes with continuous time, the values of the white noise process in a strong sense $\{\varsigma(\tau), P[\{\varsigma(0)=0\}=1]\}, \varsigma \in(-\infty, \infty)$ and those of the process with independent increments $\{\eta(t), t \in(-\infty, \infty)\}$ are connected by the equation $\eta(t)=\int_{0}^{t} \varsigma(\tau) d(\tau), \quad t \in(\infty, \infty), \varsigma(\tau)$ is stochastically equivalent to $\varsigma(-\tau)$. In our case, $\eta(t)$ is a non-homogeneous Hilbert process with independent increments. There exists a $T>0$ for the process $\eta(t)$, which is the basis for fulfillment of the following properties:

$$
\begin{gathered}
d \kappa_{1}(t)=d \kappa_{1}(t+T), d \kappa_{2}(t)=d \kappa_{2}(t+T), \\
d_{x} d_{t} L(x, t)=d_{x} d_{t} L(x, t+T), \quad \forall t \in(-\infty, \infty),
\end{gathered}
$$


where $\kappa_{1}(t)$ and $\kappa_{2}(t)$ are the first cumulant functions of the process $\eta(t)$, while $L(x, t)$ is a Poisson jump spectrum in the Levy form, $T$ is a correlation period, and $\varsigma(\tau)$ is called a periodic white noise. A logarithm of a characteristic function of the real random process $\eta(t)$ in the Levy form is

$$
\ln f_{\eta}(u, t)=\left\{i \mu(t) \varepsilon u-\frac{D^{2}(t)}{2} u^{2}+\int_{-\infty}^{\infty}\left[e^{i u \varepsilon x}-1-\frac{i u \varepsilon x}{1+x^{2}}\right] d_{x} L(x, t)\right\}, \quad t \in(-\infty, \infty),
$$

where $D^{2}(t), \mu(t)$ are some variables, $\varepsilon=\operatorname{sign} t$ and $L(x, t)$ is the Poisson jump spectrum in the Levy form.

Non-stationary linear random processes can be defined by application of nonstationary random periodic processes as with independent increments $[4,12]$

$$
\xi(t)=\int_{-\infty}^{\infty} \varphi(\tau, t) d \eta(\tau), \quad t \in(-\infty, \infty),
$$

where $\varphi(\tau, t) \in L_{2, \kappa}$ is a real nonrandom periodic numerical function on $t$ that has the property $\int_{-\infty}^{\infty} \varphi^{2}(\tau, t) d \kappa_{2}(\tau)<\infty$, for every fixed $t \in(-\infty, \infty) ;\{\eta(\tau)$, $\eta(0), t \in(-\infty, \infty)\}$ is called generating process. The properties (4) are carried out for $\eta(t)$ [11]. Generalization to the complex case proceeds in the traditional way.

Logarithms of the characteristic function of the linear random process (5) in the Levy form are represented in $[12,13]$. Random periodic processes can be widely used in many applications: the problem of detection of signals from noise, classification of information signals, and many others. Using the model one can generate pseudorandom series with the required probabilistic characteristics in infinitely divisible class of distributions.

Linear autoregressive process. The method may be used for construction of the discrete-time mathematical models of information signals. Let us consider some properties of linear processes of autoregression in greater detail. Autoregression linear processes may be set in the following way:

$$
\xi_{t}+\sum_{j=1}^{p} a_{j} \xi_{t-j}=\varsigma_{t}, t \in Z
$$

where $\left\{a_{j}, a_{j} \neq 0, j=\overline{1, p}\right\}$ are the autoregression parameters; $p$ is the autoregression order; $\left\{\zeta_{t}, t \in Z\right\}$ is the stationary random process with discrete time and independent values, which has the infinitely divisible distribution law $P\left\{\varsigma_{0}=0\right\}=1$. This process is often referred to as the generating process. It is proposed that the process $\zeta_{t}$ is stationary in the narrow sense and ergodic theorems 
are fulfilled [14]. It is also assumed that the solutions of the characteristic equation $\Psi(z)=a_{p}+a_{p-1} z+\ldots+a_{1} z^{p}$, on the complex plane lie within the unit disk [14]. Then, the difference equation (1) has the only stationary solution

$$
\xi_{t}=\sum_{\tau=0}^{\infty} \varphi(\tau) \zeta_{t-\tau}
$$

where $\{\varphi(\tau), \tau \in Z\}$ is some numerical sequence, which is referred to as a pulse-transition function or the kernel of the random process $\xi_{t}$. It is assumed that the following relationship is fulfilled

$$
\sum_{\tau=0}^{\infty} \varphi^{2}(\tau)<\infty
$$

Consequently, the autoregression linear process $\xi_{t}$ of order $p$ may be considered as the process of the sliding average infinite order. The kernel $\varphi(\tau)$ is related to the parameters of autoregression $\left\{a_{j}, j=\overline{1, p}\right\}$ [13].

Autoregression processes are widely practiced when constructing mathematical models of information signals of different types and during their analysis and synthesis. To classify such processes their energy characteristics are used very often, but in problems of classification of stochastic information signals in the case of non-Gaussian distribution, the information, which energy spectra (within the framework of the first two moments) possess is often not enough for reliable recognition and classification of such signals. Then it is expedient to use information on higher moments (integral characteristics) or statistical characteristics of distributing such signals.

Stationary autoregressive processes have found wide application in solving different radio engineering problems [13-15]. However, many processes in radiophysics, radiolocation, telemetry, hydroacoustics, meteorology, astronomy, biomedical systems and, consequently, the random functions describing such processes possess the characteristics repeating in time or space. Such processes can be used to describe, for example, signals at the mixer output, where a periodic oscillation and stationary noise are applied to the mixer inputs; the signals of parametric amplifiers with repetitive pumping, signals of nonlinear self-oscillatory systems, output signals of linear systems with cyclically varying parameters, and magnetic noises during the cyclic ferromagnetic magnetization switching. By the period of process variation is usually meant a time or space interval of complete repetition of variations of process characteristics, though the proper values (realizations) of such random process may not have the same properties. In this case for mathematical simulation it is expedient to apply the random processes with periodic structures. 
Linear autoregressive process with periodic structures. The present paper also deals with the consideration of singularities and specific properties of linear autoregressive processes with periodic structures. Such processes are the generalization of linear stationary autoregressive processes [16]. The peculiarity of the given processes is the possibility of their use for the description of non-Gaussian periodic random signals.

Real random process $\{\xi, t \in Z\}$ defined over the set of integers $Z=$ $=\{\ldots,-1,0,1, \ldots\}$ is called the autoregressive process with periodically varying autoregression parameters. It can be written as follows: $\xi_{t}+a_{1}(t-1) \xi_{t-1}+\ldots$ $\ldots+a_{p}(t-p) \xi_{t-p}=\varsigma_{t}$, where $a_{p}(t)$ are the autoregression parameters alternating in time with the same period, i.e., $a_{1}(t)=a_{1}(t+T), \ldots, a_{p}(t)=a_{p}(t+T), p>0$, $p \in Z \forall t \in Z$, is the order of autoregression, $\varsigma_{t}$ is the random process with discrete time and independent values having an infinitely divisible distribution law.

It was shown in [16] that the Hilbert autoregressive process with cyclically time varying autoregression parameters having the same period $T>0$ generated by a random process with independent values and completely divisible distribution law represents a linear random process with discrete time and periodic kernel (in terms of $t$ ).

Let us consider a linear autoregressive process having the periodic structure of the generating process. Such process satisfies the following difference equation: $\xi_{t}+a_{1} \xi_{t-1}+\ldots+a_{t-p}=\varsigma_{t}, t \in Z$, where $\left\{a_{j}, j=\overline{1, p}\right\}$ are the autoregression parameters, $p$ is the order of autoregression, $\varsigma_{t}$ is the generating process having the properties presented below.

Let us assume that $\eta_{t}=\varsigma_{t}-\varsigma_{t-1}, t \in Z$ is the first difference of generating process $\varsigma_{t}$. Let us also assume the existence of such $T>0$ that for all $\tau$ and $t$ the following relationships are fulfilled:

$$
\kappa_{1}(\tau)=\kappa_{1}(\tau+T) ; \kappa_{2}(\tau)=\kappa_{2}(\tau+T), d_{x} L(x, \tau)=d_{x} L(x, \tau+T),
$$

where $\kappa_{1}(\tau)$ and $\kappa_{2}(\tau)$ are the first cumulant functions of the process $\eta_{t} ; L(x, \tau)$ is the Poisson spectrum of jumps in the Levi formula for the process $\zeta_{t}$. It was shown in [14] that the linear autoregressive process with periodic generating process is a periodic random process in a strict sense.

Conclusion. The above considered models of random processes can be applied for the simulation of information signals of expert systems different types, various cyclically changing radiophysical processes, random signals in biomedical investigations, etc., $t \in Z$.

The autoregressive processes with cyclically time-varying autoregression parameters having the same period and a periodic generating process can be also referred to periodic random processes in strict sense. The method could be applied to develop other types of mathematical models such as considered in the papers [17-18]. 


\section{REFERENCES}

1. Ito, K. (1954), Stationary random distribution, Mem. Coll. Univ., Vol. 28, pp.209-223.

2. Kolmogorov, A. (1932), Sulla forma generale di un processo stocastieo omegeneo, Atti. della Reale Academia nazionale dei Lincei. Ser. sesta Rendiconti, Vol. 15, no.10, pp. 805808.

3. Khinchin, A. (1938), Predelnye zakony dlya sum nezavisimykh stokhasticheskikh velichin [Limit laws for sums of independent stochastic values], ONTI, Moscow-Leningrad, USSR.

4. Martchenko, B. (1973), Metod stokhasticheskikh integralnykh predstavleniyi i yego primeneniye $v$ radiotekhnike [Method of stochastic integral representations and its applications in radio-engineering], Naukova dumka, Kiev, USSR.

5. Brockwell, P.J. and Davis, R.A. (2002), Introduction to time series and forecasting, 2nd edition, Springer, New York, USA.

6. Iwueze, I.S., Arimie, C.O., Iwu, H.C. and Onyemachi, E. (2017), Some applications of the linear Gaussian white noise process, Applied Mathematics, Vol. 8, pp. 1918-1938.

7. Zvaritch, V., Myslovitch, M. and Martchenko, B. (1994), White noise in information signals models, Appl. Math. Lett., Vol. 7, no. 3, pp. 93-95.

8. Ogura, H. (1971), Spectral representation of a periodic nonstationary random process, IEEE Transaction of Information Theory, Vol. IT-17, no. 2, pp. 143-149.

9. Skhorokhod, A. (1964), Sluchainye protsessy c nezavisimymi prirashcheniyami [Random processes with independent increments], Nauka, Moscow, USSR.

10. Slutskiy, E.E. (1960), Izbrannye statyi [Selected papers], Izdatelstvo Academii Nauk SSSR, Moscow, USSR.

11. Zvaritch, V., Myslovitch, M. and Martchenko, B. (1995), The models of random periodic information signals on the white noise bases, Appl. Math. Lett., Vol. 8, no. 3, pp. 87-89.

12. Zvaritch, V., Myslovitch, M. and Martchenko, B. (1995), Stochastically periodical random processes used as models of information signals, Radioelectronics and Communication Systems, Vol. 38, no. 1, pp. 129-132.

13. Zvarich, V.N. and Marchenko, B.G. (1999), "The method of determining characteristic functions of the generating processes for linear autoregression processes", Izvestiya Vysshikh Uchebnykh Zavedeniy Radioelektroniki, Vol. 42, no. 7, pp. 64-71.

14. Zvarich, V.N. and Marchenko, B.G. (2002), "Characteristic function of the generating process in the model of stationary linear AR-gamma process", Izvestiya Vysshikh Uchebnykh Zavedeniy Radioelektroniki, Vol. 45, no. 8, pp. 12-18.

15. Zvarich, V.N. (2016), Peculiarities of finding characteristic functions of generating process in the model of stationary linear AR(2) process with negative binomial distribution, Radioelectronics and Communication Systems, Vol. 59, no. 12, pp. 567-573.

16. Zvarich, V.N. and Marchenko, B.G. (2011), Linear autoregressive processes with periodic structures as models of information signals, Radioelectronics and Communication Systems, Vol. 54, no. 7, pp. 367-372.

17. Shao, X. (2011), Testing for white noise under unknown dependence and its applications to diagnostic checking for time series models, Econometric Theory, Vol. 27, no. 2, pp. 312-343.

18. Zvaritch, V. and Glazkova, E. (2015), Some singularities of kernels of linear AR and ARMA processes and their applications to simulation of information signals, Computational Problems of Electrical Engineering, Vol. 5, no. 1, pp. 71-74.

Received 16.02.18 


\section{В.М. Зварич, М.В. Мислович}

\section{БІЛИЙ ШУМ В ДЕЯКИХ ЗАДАЧАХ МОДЕЛЮВАННЯ ІНФОРМАЦІЙНИХ СИГНАЛІВ}

Запропоновано конструктивний метод завдання математичних моделей інформаційних сигналів на основі білих шумів. Як приклад побудови математичних моделей інформаційних сигналів розглянуто лінійні випадкові процеси, лінійні випадкові процеси 3 періодичними структурами, лінійні процеси авторегресії, лінійні процеси авторегресії 3 періодичними структурами.

Клю ч о в $і$ сл о в а: білий шум, лінійний випадковий прочес, лінійні випадкові процеси з періодичними структурами, лінійні процеси авторегресії.

\section{В.Н. Зварич, М.В. Мислович}

\section{БЕЛЫЙ ШУМ В НЕКОТОРЫХ ЗАДАЧАХ МОДЕЛИРОВАНИЯ ИНФОРМАЦИОННЫХ СИГНАЛОВ}

Предложен конструктивный метод задания математических моделей информационных сигналов на основе белых шумов. В качестве примера построения математических моделей рассмотрены линейные случайные процессы, линейные случайные процессы с периодическими структурами, линейные процессы авторегресии, линейные процессы авторегрессии с периодическими структурами.

Ключ ю в ы е сло в а: бельй шум, линейный случайный процесс, линейные случайныле проиессы с периодическими структурами, линейные проиессы авторегресии.

ZVARITCH Valerij Nikolayevich, Doctor of sciences (engineering), leading scientific worker of the Institute of Electrodynamics, NAS of Ukraine, graduated from the National Technical University of Ukraine «Kiev Polytechnic Institute» in 1982. Sphere of scientific research: modeling of information signals with the use of statistical approach, development of computer systems of vibrodiagnostics.

MYSLOVICH Mikhail Vladimirovich, Doctor of sciences (engineering), professor, head of the department of the Institute of Electrodynamics, NAS of Ukraine, graduated from the National Technical University of Ukraine «Kiev Polytechnic Institute» in 1975. Sphere of scientific research: mathematical modeling, technical diagnostics, mathematical statistics, processing of signals. 